\title{
Brain Natriuretic Peptide Levels in Acute Inferior Myocardial Infarction
}

\author{
Tarik Yildirim ${ }^{\mathrm{a}, \mathrm{d}}$, Seda Elcim Yildirim ${ }^{\mathrm{a}}$, Meryem Aktoz ${ }^{\mathrm{b}}$, Armagan Altun ${ }^{\mathrm{c}}$
}

\begin{abstract}
Background: Our objective was to evaluate the relationship between initial serum brain natriuretic peptide (BNP) levels and right ventricular functions in inferior myocardial infarction (MI) with and without right ventricular involvement.
\end{abstract}

Methods: The study included 61 patients, who presented with acute inferior MI. Twenty-seven patients had right ventricular involvement. Blood samples for BNP were obtained from each patient on admission. Echocardiographic assessments were performed and recorded during the first $12 \mathrm{~h}$. Right ventricular involvement was determined by electrocardiography, conventional and tissue Doppler echocardiography (TDI).

Results: In inferior MI with right ventricular involvement, tricuspid annulus planimetric systolic excursion (TAPSE) and right ventricular fractional area change were lower, and left ventricular E/E' ratio was higher. In the group with BNP levels above $400 \mathrm{pg} / \mathrm{mL}$, left ventricular end-diastolic diameter and left ventricular endsystolic diameter were higher, and left ventricular ejection fraction and TAPSE, indicator of right ventricular systolic function, were lower. The elevated BNP levels were negatively correlated with RSm and TAPSE, while they were positively correlated with the $\mathrm{E} / \mathrm{E}$ ' ratio. The systolic blood pressure and left ventricular enddiastolic diameter during admission were independent predictors of BNP levels.

Conclusions: In acute inferior MI, initially increased BNP levels may be valuable in predicting the right ventricle involvement. Higher rates of hypotension, right ventricular dysfunction and increased left ventricle diameters are observed in patients with BNP levels $\geq 400 \mathrm{pg}$ / $\mathrm{mL}$.

Keywords: BNP; Doppler echocardiography; Inferior myocardial in-

Manuscript submitted December 15, 2017, accepted January 3, 2018

aDepartment of Cardiology, Mugla Sitki Kocman University, Mugla, Turkey bepartment of Cardiology, Trakya University Faculty of Medicine, Edirne, Turkey

'Department of Cardiology, Baskent University Faculty of Medicine, Istanbul, Turkey

${ }^{\mathrm{d} C}$ Corresponding Author: Tarik Yildirim, Department of Cardiology, Mugla Sitki Kocman University, Mugla, Turkey. Email: kdrtarik@gmail.com

doi: https://doi.org/10.14740/jocmr3324w farction; Right ventricle

\section{Introduction}

Both systolic and diastolic left ventricular functions are impaired following acute myocardial infarction (AMI). The extent of impairment in left ventricular function is one of the most important factors of morbidity and mortality [1]. In cases of myocardial infarction (MI) associated with left ventricular dysfunction, the right ventricular dysfunction is an independent predictor of mortality [2]. Approximately $50 \%$ of acute inferior MIs are accompanied by right ventricular myocardial infarction (RVMI) [3]. RVMI is associated with suppression of the right ventricular functions, resulting in right heart failure and decreased heart rate. Although elevated jugular venous pressure, clear lung fields and hypotension are specific to RVMI, their sensitivity is less than 25\% [4]. ST segment elevation in the right precordial derivation V4 (V4R) indicates RVMI, but it has a disadvantage of being temporary [5]. The brain natriuretic peptide (BNP) is synthesized from the ventricular myocardium as a result of increased cardiac wall stress due to pressure overload or volume expansion. BNP can decrease right atrial pressure, systemic vascular resistance, stimulation of sympathetic nerves, aldosterone secretion and cell hypertrophy while increasing the excretion of sodium [6]. Elevated BNP levels were found to be poor outcome in heart failure, and in patients who had a first AMI and underwent primary percutaneous transluminal coronary angioplasty (PTCA), elevated BNP levels was found to be related with poor outcome [7]. In the present study, our objective was to evaluate the relationship of right ventricular dysfunction as determined by conventional and tissue Doppler echocardiography (TDI) with the serum BNP levels in inferior MI with and without right ventricular involvement.

\section{Materials and Methods}

This prospective study was developed and adapted in accordance with the guidelines and ethical principles defined in the Declaration of Helsinki and all patients approved and consented to participate in the study. We enrolled 61 patients who had a first acute inferior MI with/without right ventricular involvement with a normal sinus rhythm and a heart rate 
Table 1. Baseline Characteristics of Patients

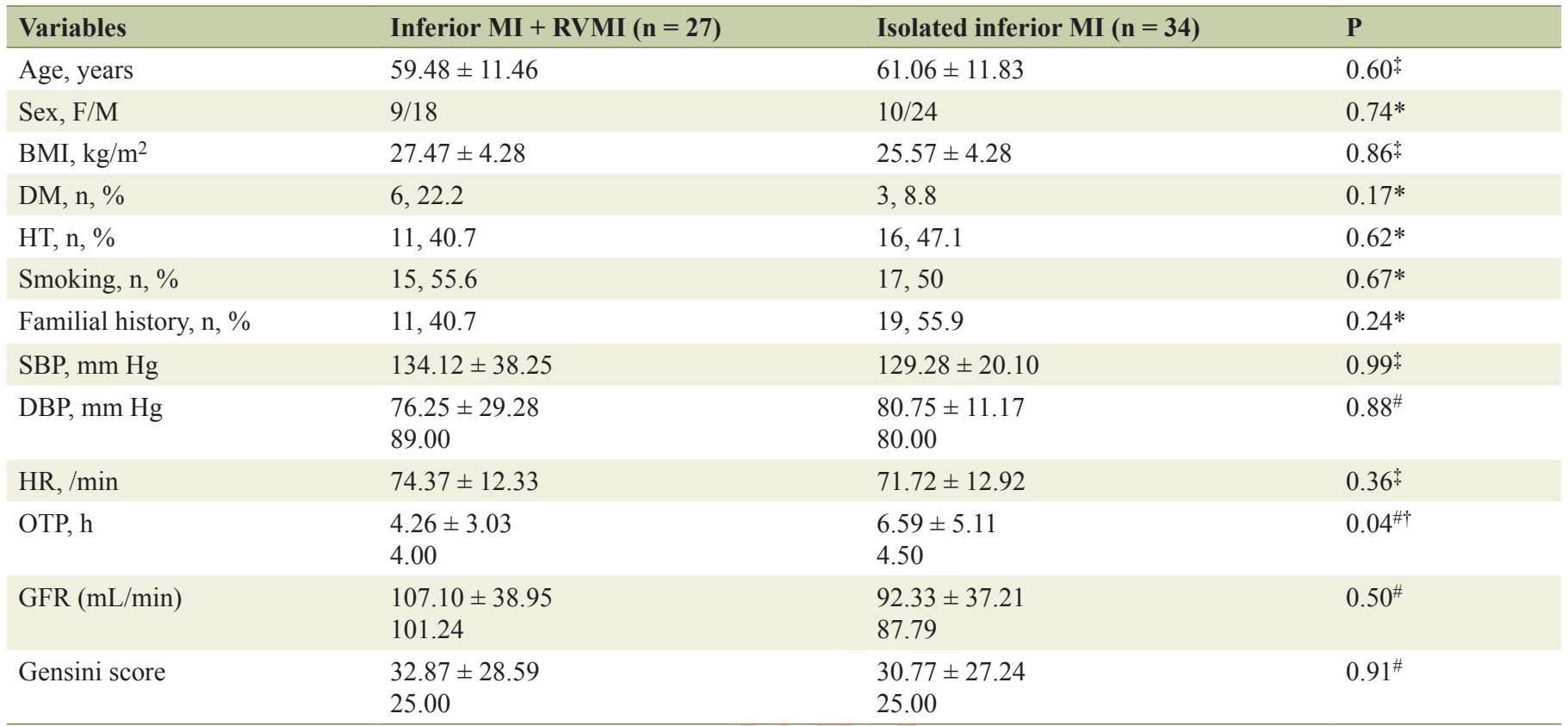

BMI: body mass index; DBP: diastolic blood pressure; DM: diabetes mellitus; GFR: glomerular filtration rate; HR: heart rate; HT: hypertension; OTP: onset time of chest pain; SBP: systolic blood pressure. ${ }^{\ddagger}$ Independent groups $t$-test, \#Mann-Whitney $U$ test, ${ }^{\dagger}$ Significant at $p<0.05$. Data are shown in mean \pm standard deviation, median or number (\%).

ranging from 50 to $120 \mathrm{~min}$. The exclusion criteria were: prior MI and/or revascularization, mild and severe valvular heart disease, pericardial disease, pulmonary hypertension, patients with rhythm other than normal sinus rhythm and chronic kidney disease. There were 34 patients with isolated acute inferior MI (24 males and 10 females), and 27 patients with right ventricular involvement (18 males and nine females). Sixteen patients were excluded because of atrial fibrillation, prior MI and severe mitral regurgitation. The diagnostic criteria of MI were as follows: A rise and/or fall of cardiac troponin levels at least one value above the 99 percentile and ischemic symptoms, ST/T changes, new left bundle branch block, new pathological Q waves, echocardiographic evidence of new regional wall motion abnormality or detection of intracoronary thrombus by angiography or autopsy [8]. An ST segment elevation on admission equal to or greater than $1 \mathrm{~mm}$ in RV4 was used as a diagnostic criterion for RVMI. ST elevation on admission was present in all patients. Twenty-seven patients (79.4\%) were given thrombolytic agents in inferior MI. Seven patients $(20.6 \%)$ underwent primary percutaneous coronary intervention. Twenty-one patients $(77.8 \%)$ were given thrombolytic agents in inferior MI with right ventricular involvement and six patients $(22.2 \%)$ underwent primary percutaneous coronary intervention. After admission of the patients who presented with acute inferior MI, blood samples were drawn into 5-mL EDTA tubes during the first $12 \mathrm{~h}$. Serum BNP levels were detected by radioimmunoassay using a BNP RIA kit (Phoenix Pharmaceuticals, CA, USA) and a DPC Gambyt-CR Gamma Counter (DPC Diagnostic Products Corporation, Los Angeles, CA, USA). The cutoff BNP value was $100 \mathrm{pg} / \mathrm{mL}$. Serum troponin I (TnI) levels were detected by fluorometry us- ing a Siemens troponin kit (cTnI direct fluorometric, Siemens, New York, USA). The normal value was $0.00-0.06 \mathrm{ng} / \mathrm{mL}$.

The patients underwent echocardiographic evaluation during the first $12 \mathrm{~h}$ after admission. After ECG monitorization, an echocardiographic evaluation was performed from parasternal long axis, parasternal short axis, apical four chamber, and subcostal views using an echocardiographic unit (GE Vingmed Ultrasound, Horten, Norway) and 2.5 - 3.5 MHz transducers in the left lateral decubitus position. The patients underwent Mmode, two-dimensional echocardiography, pulse wave (PW) and continuous wave (CW) Doppler and colored tissue Doppler echocardiographic evaluation. All Doppler measurements were recorded at the end of the expirium while patients were holding their breath. An average of three consecutive measurements were calculated. An echocardiographic evaluation was performed using the parasternal long axis, short axis, and apical four- and two-chamber images to analyze the left ventricular functions. Following the evaluation of aortic and left ventricular wall thickness, the left ventricular ejection fraction (LVEF) was calculated by the Teicholz method. Wall motion was evaluated from the parasternal short axis view. Biplane ejection fraction was determined from the apical four-chamber and two-chamber views using the Simpson method. Sample volume was placed on the ventricular surface of the mitral valve to evaluate diastolic functions. Right ventricle fractional area change (RVFAC), tricuspid annular plane systolic excursion (TAPSE), and right ventricular systolic wave (RSm) were used to evaluate right ventricular systolic functions, and right ventricle myocardial performance index (RVMPI), tissue Doppler imaging for global functions. We compared the groups with and without right ventricular involvement. After this as- 
Table 2. Left Ventricular Echocardiographic Parameters

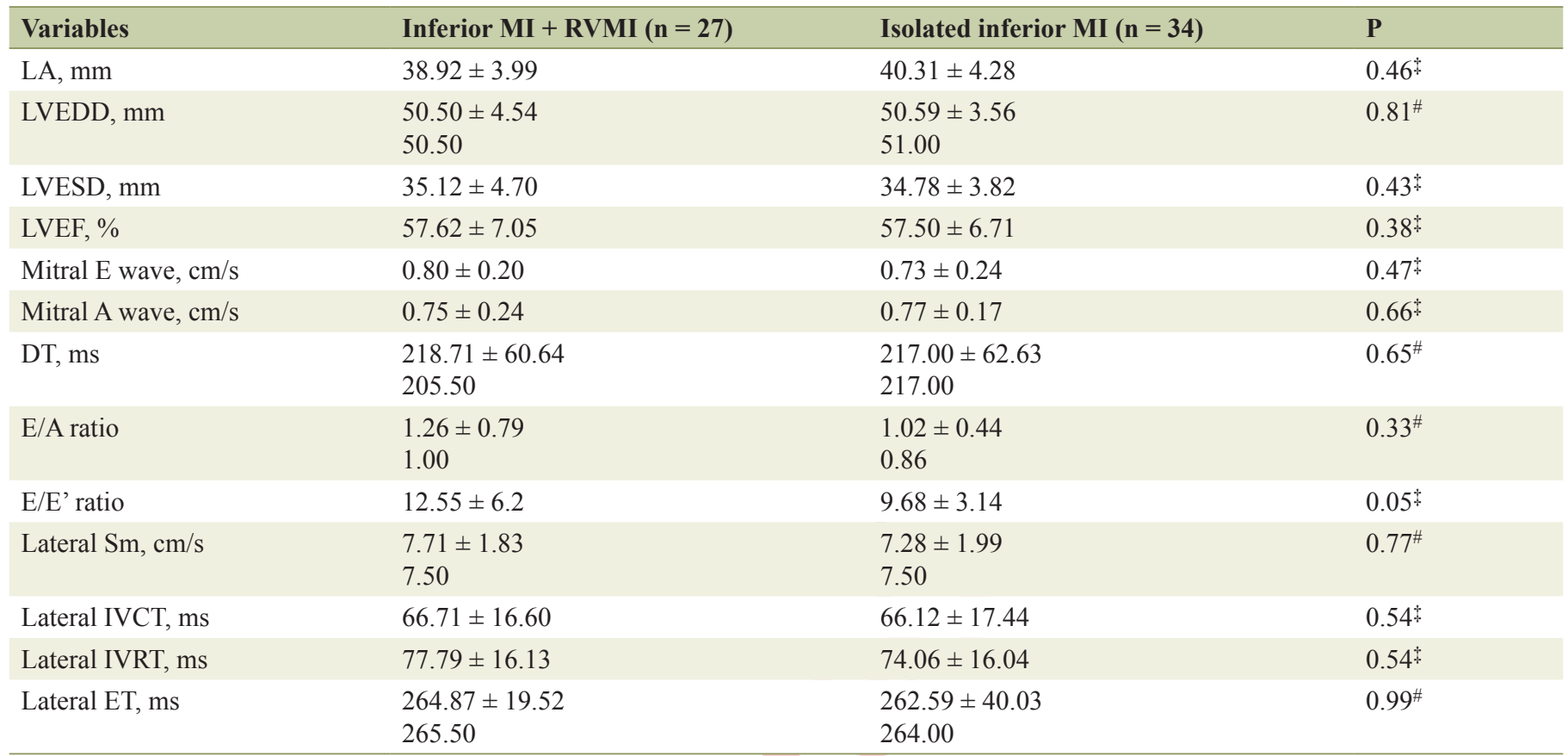

DT: deceleration time; E/A ratio: ratio of early to late diastolic filling; E/E': ratio of early filling velocity to tissue Doppler early filling velocity; ET: ejection time; IVCT: isovolumetric contraction time; IVRT: isovolumetric relaxation time; LA: left atrium; LVEDD: left ventricular end-diastolic diameter; LVEF: left ventricular ejection fraction; LVESD: left ventricular end-systolic diameter; RVMI: right ventricular myocardial infarction. ${ }^{\ddagger}$ Independent groups $t$ test. \#Mann-Whitney $U$ test. Data are shown in mean \pm standard deviation, and median.

sessment, we compared the groups according to BNP levels.

\section{Statistical analysis}

Statistical analysis was performed using the STATISTICAAXA 7.1 Serial No. AXA 507C775506FAN3 (StatSoft Inc, Tulsa, USA) and MedCalc 12.3.0.0 statistical softwares (MedCalc Software bvba, Ostend, Belgium). After testing for normal distribution of measurable data by Shapiro-Wilk test, we used independent groups $t$-test for intergroup comparisons for those with a normal distribution, and Mann-Whitney U test for those without a normal distribution. Pearson correlations were used to analyze the relationship between variables. For qualitative data, we used Pearson's Chi-squared test, Fisher's exact test, Yatescorrected Chi-square test and two-sample Kolmogrov-Smirnov test. Descriptive statistics were expressed as median values and arithmetic mean \pm standard deviation. For all statistical analyses, a P-value $<0.05$ was considered statistically significant.

\section{Results}

The study included a total of 61 patients. Of these patients, $27(44 \%)$ had acute inferior MI with right ventricular involvement (mean age, $59.48 \pm 11.46$ years) and $34(56 \%)$ had acute inferior MI (mean age, $61.06 \pm 11.83$ years), and $42(69 \%)$ were males, and 19 (31\%) were females. As shown in Table 1 , no significant difference was found between two groups in baseline characteristics except for onset time of chest pain.

\section{Echocardiographic parameters}

Table 2 shows left ventricular echocardiographic parameters. There was no significant difference except E/E' ratio, which is an indicator of diastolic dysfunction. Right ventricular echocardiographic parameters are shown in Table 3. RVFAC and TAPSE were significantly lower in patients with inferior MI with right ventricular involvement. No significant difference was found in groups between BNP levels. The TnI levels were higher in patients who presented with isolated inferior MI (P $=0.01$ ) (Table 4). When patients were divided into two groups according to BNP levels, left ventricular end-diastolic diameter (LVEDD) and left ventricular end-systolic diameter (LVESD) were higher, and LVEF was lower in patients with BNP level $\geq 400 \mathrm{pg} / \mathrm{mL}$. The right atrial diameter was larger and TAPSE was lower in the group with BNP level $\geq 400 \mathrm{pg} / \mathrm{mL}$ (Table 5). Correlation analysis between BNP levels and echocardiographic parameters showed a relationship between the BNP levels and the RSm, TAPSE and left ventricular mass index (LVMI) (Table 6). The systolic blood pressure and left venticular end-diastolic diameter are independent predictors of BNP levels (Table 7).

\section{Discussion}

In this study, we found that RVFAC and TAPSE were lower in 
Table 3. Right Ventricular Echocardiographic Parameters

\begin{tabular}{llll}
\hline Variables & Inferior MI + RVMI $(\mathbf{n = 2 7 )}$ & Isolated inferior MI $(\mathbf{n}=\mathbf{3 4})$ & P \\
\hline RSm, cm $/ \mathrm{s}$ & $11.00 \pm 1.50$ & $13.94 \pm 2.17$ & $0.90^{\#}$ \\
& 13.00 & 12.50 & $0.62^{\#}$ \\
RVMPI & $0.58 \pm 0.14$ & $0.61 \pm 0.16$ & 0.57 \\
TAPSE, mm & 0.57 & $21.23 \pm 2.35$ & $0.00^{\# !}$ \\
RVFAC, \% & $17.04 \pm 1.64$ & 21.00 & $0.00^{\ddagger !}$ \\
\hline
\end{tabular}

RSm: right ventricular systolic wave; RVFAC: right ventricle fractional area change; RVMI: right ventricular myocardial infarction; RVMPI: right ventricular myocardial performance index; TAPSE: tricuspid annular plane systolic excursion. \#Independent groups $t$-test. \#Mann-Whitney $U$ test. !Significant at $\mathrm{P}<0.05$. Data are shown in mean \pm standard deviation, and median.

Table 4. Brain Natriuretic Peptide Levels

\begin{tabular}{llll}
\hline Variables & Inferior MI + RVMI $(\mathbf{n = 2 7 )}$ & Isolated inferior MI $(\mathbf{n}=\mathbf{3 4})$ & P \\
\hline BNP, pg/mL & $372.92 \pm 324.75$ & $430.13 \pm 320.39$ & $0.52^{\#}$ \\
TnI, ng/mL & 245.19 & 281.66 & $0.01^{\#}$ \\
& $1.78 \pm 6.13$ & $7.11 \pm 13.21$ & 1.38 \\
\hline
\end{tabular}

Data are shown as median \pm standard deviation, and median. BNP: brain natriuretic peptide; RVMI: right ventricular myocardial infarction; Tnl: troponin I. \#Mann-Whitney U test.

patients with inferior MI with right ventricular involvement. In the BNP level $\geq 400 \mathrm{pg} / \mathrm{mL}$ group, LVEDD and LVESD were higher, LVEF and TAPSE were lower and RA diameter was larger than BNP level $<400 \mathrm{pg} / \mathrm{mL}$ group. The systolic blood pressure and left venticular end-diastolic diameter are independent predictors of BNP levels. The incidence of acute inferior MI accompanied with right ventricular involvement is ranging from $10 \%$ to $50 \%[9,10]$. The incidence of death, shock and arrhyth-

Table 5. Echocardiographic Parameters With BNP Levels

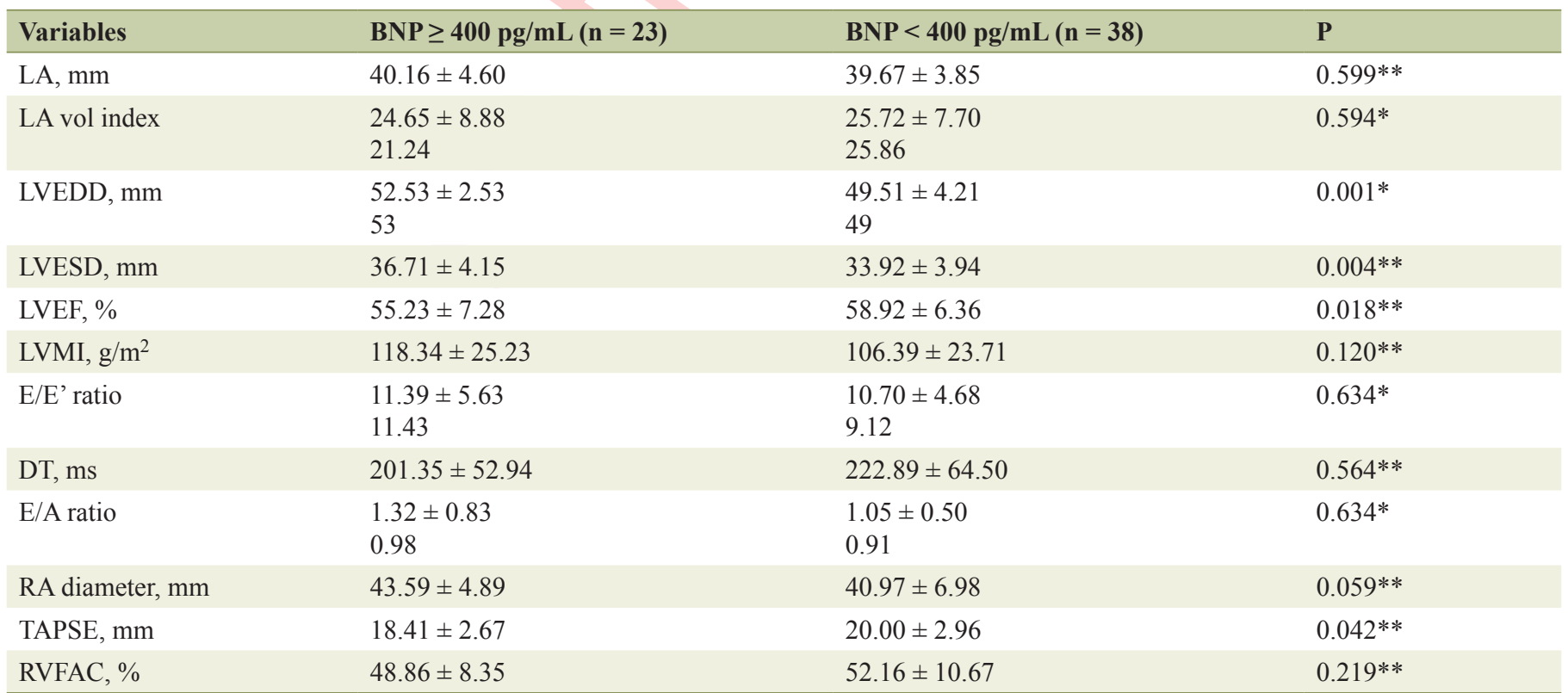

Data are shown as median \pm standard deviation, and median. BNP: brain natriuretic peptide; DT: deceleration time; E/A: ratio of early to late diastolic filling; E/E': ratio of early mitral inflow filling to tissue Doppler early filling velocity; LA: left atrium; LA vol index: left atrial volume index; LVEDD: left ventricular end-diastolic diameter; LVEF: left ventricular ejection fraction; LVESD: left ventricular end -systolic diameter; LVMI: left ventricular mass index; RA: right atrium; RVFAC: right ventricular fractional area change; TAPSE: tricuspid annular plane systolic excursion. *Mann-Whitney U test. **Independent groups $t$-test. 
Table 6. Correlation Between Brain Natriuretic Peptide Levels and Echocardiographic Parameters

\begin{tabular}{lll} 
Variables & Correlation level & $\mathbf{P}$ \\
\hline RSm & $-0.347^{\mathrm{x}}$ & $0.045^{!}$ \\
TAPSE & $-0.402^{\mathrm{x}}$ & $0.018^{!}$ \\
LVMI & $0.353^{\mathrm{x}}$ & $0.048^{!}$ \\
\hline
\end{tabular}

LVMI: left ventricular mass index; RSm: tricuspid lateral annulus $\mathrm{S}$ wave; TAPSE: tricuspid annular plane systolic excursion. ${ }^{\text {PPearson's }}$ correlation analysis. 'Significant at $<0.05$.

mia is higher in patients with right ventricular involvement [2, 3]. In V4R ST elevation has a sensitivity of $88 \%$, specificity of $78 \%$ in the diagnosis of RVMI [11]. Because this is a temporary finding, some patients with acute inferior MI might actually have a nondiagnosed RVMI. BNP is secreted from the ventricles in response to volume expansion or increased pressure load. The BNP level increases in 2 - $4 \mathrm{~h}$ after the onset of ischemic symptoms, with a tendency to stabilize over the first $24 \mathrm{~h}$ [6]. In the present study, no significant difference was found in BNP level between inferior MI with right ventricular involvement and isolated inferior MI groups. The most important factor that caused this finding was the time difference between onset of symptoms and hospital admission. Serum BNP levels may be varied substantially during this time period. Although BNP levels were higher in patients with inferior MI and right ventricular involvement in a study by Kaya et al, in our study there was no difference in BNP levels since patients with right ventricular involvement were more symptomatic, and they presented earlier [12]. Studies in patients with unstable angina pectoris and MI have demonstrated the prognostic value of BNP along with troponin level [13-15]. In the present study, TnI levels were significantly higher in the group with isolated inferior MI during admission compared to the group with right ventricular involvement. We considered that lower TnI levels in patients with RVMI were associated with the fact that the patients were more symptomatic, and they presented earlier without giving any chance to an increase in TnI level. A study compared BNP levels of patients with NYHA class 2 and 3 heart failure and LVEF of $<45 \%$, and they found a negative correlation between BNP levels and LVEF [16]. Another study showed that BNP levels were higher in patients with right ventricular $\mathrm{EF}<40 \%$ compared to patients with $\mathrm{EF}>40 \%$ [17]. We found no correlation between BNP levels and LVEF. However, the higher BNP group had higher LVEDD and LVMI. In addition to that, TAPSE and RSm values were lower. We also found right ventricular systolic dysfunction in inferior MI with right ventricular involvement (TAPSE, RSm, and RVFAC).

Skrzypek et al showed that there was a significant cor- relation between systolic and diastolic dysfunction with the levels of $\mathrm{N}$ terminal probrain natriuretic peptide (NT-proBNP) [18]. Besides another study comparing BNP levels with left ventricular diastolic function parameters, Ono et al showed that mitral inflows ( $\mathrm{E}$ and $\mathrm{A}$ waves) were correlated with BNP levels [19]. And also, they found no significant relationship between BNP levels and isovolumetric relaxation time (IVRT), the earliest abnormality parameter of diastolic function. Similarly, we found no relationship between BNP levels and diastolic dysfunction. In a study by Nagaya et al which compared right ventricular dysfunctions and BNP levels in patients with pulmonary hypertension, there was a relationship between right ventricular functions and BNP levels, while no relationship was found between right ventricular diastolic diameter and BNP levels [20]. It may be attributed to the higher pulmonary arterial pressure in these patients. In the present study, there was no significant difference in right ventricular global functions between groups with higher and lower BNP levels. Unlike the study by Nagaya et al, our patients had no pathology of pulmonary hypertension, which contributed to the right ventricular dysfunction. We observed no difference in right ventricular diastolic diameter between groups with higher and lower BNP level. The systolic and diastolic blood pressures were lower in our group with BNP level $\geq 400 \mathrm{pg}$ / $\mathrm{mL}$. We thought that this reduction might be associated with right ventricular MI. Mayer et al evaluated the relationship of BNP levels with LVEF, left atrial diameter, LVEDD, posterior wall and septum thickness in heart failure patients with functional mitral regurgitation. Consequently, they found a positive correlation between LVEDD and BNP levels [21]. In the group with BNP level $\geq 400 \mathrm{pg} / \mathrm{mL}$, we determined $33.3 \%$ right ventricular involvement and in the group with BNP level $<400 \mathrm{pg} / \mathrm{mL}$ we found right ventricular involvement $66.6 \%$. In the present study, LVEDD and lvesd were larger, and LVEF and TAPSE were lower in patients with inferior MI with and without right ventricular involvement and BNP level $\geq 400 \mathrm{pg} /$ $\mathrm{mL}$ like as shown in the study which has been presented by Radwan et al [22].

In our study, we concluded that initial BNP levels $\geq 400$ $\mathrm{pg} / \mathrm{mL}$ in patients presenting with acute inferior MI might predict right ventricular dysfunction. In addition, higher rates of hypotension, right ventricular dysfunction and increase in left ventricule diameters were observed in patients with BNP levels $\geq 400 \mathrm{pg} / \mathrm{mL}$.

\section{Study limitations}

Evaluation of right ventricular function only by echocardiography is the first limiting factor of this study. Right ventricular

Table 7. Stepwise Logistic Regression Analysis for Brain Natiuretic Peptide Levels

\begin{tabular}{llllll} 
Variables & B & Odds ratio & \multicolumn{3}{c}{ 95\% confidence interval } \\
\cline { 3 - 6 } & & & Lower limit & Upper limit & P \\
SBP & -0.029 & 0.972 & 0.947 & 0.996 & 0.025 \\
LVEDD & 0.281 & 1.324 & 1.090 & 1.607 & 0.005 \\
\hline
\end{tabular}

SBP: systolic blood pressure; LVEDD: left ventricular end-diastolic diameter. 
functions can also be evaluated by magnetic resonance and nuclear imaging modalities. However, their high cost, risks posed to the patients as well as unavailability of them as a bedside modality, restricts their routine use. The second limiting factor is the diagnosis of RVMI only with RV4 lead ST elevation which is temporary. Thrombolytic treatment rather than primary percutaneous coronary intervention was preferred treatment modality in our clinic during this study. This is another limitation of this study.

\section{Conflict of Interest}

None.

\section{References}

1. Gaudron P, Eilles C, Kugler I, Ertl G. Progressive left ventricular dysfunction and remodeling after myocardial infarction. Potential mechanisms and early predictors. Circulation. 1993;87(3):755-763.

2. Zornoff LA, Skali H, Pfeffer MA, St John Sutton M, Rouleau JL, Lamas GA, Plappert T, et al. Right ventricular dysfunction and risk of heart failure and mortality after myocardial infarction. J Am Coll Cardiol. 2002;39(9):1450-1455.

3. Kinch JW, Ryan TJ. Right ventricular infarction. N Engl J Med. 1994;330(17):1211-1217.

4. Dell'Italia LJ, Starling MR, O'Rourke RA. Physical examination for exclusion of hemodynamically important right ventricular infarction. Ann Intern Med. 1983;99(5):608-611.

5. Braat SH, Brugada P, de Zwaan C, Coenegracht JM, Wellens HJ. Value of electrocardiogram in diagnosing right ventricular involvement in patients with an acute inferior wall myocardial infarction. $\mathrm{Br}$ Heart $\mathrm{J}$. 1983;49(4):368-372.

6. Daniels LB, Maisel AS. Natriuretic peptides. J Am Coll Cardiol. 2007;50(25):2357-2368.

7. Kuklinska AM, Sobkowicz B, Mroczko B, Sawicki R, Musial WJ, Knapp M, Dobrzycki S, et al. Prognostic significance of the admission plasma B-type natriuretic peptide measurement in patients with first ST-elevation myocardial infarction in comparison with $\mathrm{C}$-reactive protein and TIMI risk score. Clin Chim Acta. 2007;382(12):106-111.

8. Thygesen K, Alpert JS, Jaffe AS, Simoons ML, Chaitman BR, White HD, Joint ESCAAHAWHFTFfUDoMI, et al. Third universal definition of myocardial infarction. J Am Coll Cardiol. 2012;60(16):1581-1598.

9. GISSI-3: effects of lisinopril and transdermal glyceryl trinitrate singly and together on 6-week mortality and ventricular function after acute myocardial infarction. Gruppo Italiano per lo Studio della Sopravvivenza nell'infarto Miocardico. Lancet. 1994;343(8906):11151122.

10. Spencer FA, Allegrone J, Goldberg RJ, Gore JM, Fox KA, Granger CB, Mehta RH, et al. Association of statin therapy with outcomes of acute coronary syndromes: the GRACE study. Ann Intern Med. 2004;140(11):857-866.

11. Somers MP, Brady WJ, Bateman DC, Mattu A, Perron AD. Additional electrocardiographic leads in the ED chest pain patient: right ventricular and posterior leads. Am J Emerg Med. 2003;21(7):563-573.

12. Kaya MG, Ozdogru I, Kalay N, Dogan A, Inanc T, Gul I, Gunebakmaz O, et al. Plasma B-type natriuretic peptide in diagnosing inferior myocardial infarction with right ventricular involvement. Coron Artery Dis. 2008;19(8):609613.

13. de Lemos JA, Morrow DA, Bentley JH, Omland T, Sabatine $\mathrm{MS}$, McCabe $\mathrm{CH}$, Hall $\mathrm{C}$, et al. The prognostic value of B-type natriuretic peptide in patients with acute coronary syndromes. N Engl J Med. 2001;345(14):10141021.

14. Lindahl B, Lindback J, Jernberg T, Johnston N, Stridsberg $\mathrm{M}$, Venge $\mathrm{P}$, Wallentin L. Serial analyses of N-terminal pro-B-type natriuretic peptide in patients with non-STsegment elevation acute coronary syndromes: a Fragmin and fast Revascularisation during In Stability in Coronary artery disease (FRISC)-II substudy. J Am Coll Cardiol. 2005;45(4):533-541.

15. Katlandur H, Kalay N, Y1lmaz Y, Gur M, Sarli B, Oguz F, Kasapkara A, et al. Relationship between B-type natriuretic peptide and coronary flow in patents with myocardial infarction of ST segment elevation receiving thrombolytc therapy. Eur J Health Sci. 2015;1:54-57.

16. Kjaer A, Hildebrandt P, Appel J, Petersen CL. Neurohormones as markers of right- and left-sided cardiac dimensions and function in patients with untreated chronic heart failure. Int J Cardiol. 2005;99(2):301-306.

17. Mariano-Goulart D, Eberle MC, Boudousq V, HejaziMoughari A, Piot C, Caderas de Kerleau C, Verdier R, et al. Major increase in brain natriuretic peptide indicates right ventricular systolic dysfunction in patients with heart failure. Eur J Heart Fail. 2003;5(4):481-488.

18. Skrzypek A, Nessler P. Asymptomatc ischemic heart dysfuncton, echocardiographic changes and NT-proBNP during 2-years observaton. Przeql Lek. 2014;71:378-383.

19. Ono M, Tanabe K, Asanuma T, Yoshitomi H, Shimizu H, Ohta Y, Shimada T. Doppler echocardiography-derived index of myocardial performance (TEI index): comparison with brain natriuretic peptide levels in various heart disease. Jpn Circ J. 2001;65(7):637-642.

20. Nagaya N, Nishikimi T, Okano Y, Uematsu M, Satoh T, Kyotani S, Kuribayashi S, et al. Plasma brain natriuretic peptide levels increase in proportion to the extent of right ventricular dysfunction in pulmonary hypertension. J Am Coll Cardiol. 1998;31(1):202-208.

21. Mayer SA, De Lemos JA, Murphy SA, Brooks S, Roberts BJ, Grayburn PA. Comparison of B-type natriuretic peptide levels in patients with heart failure with versus without mitral regurgitation. Am J Cardiol. 2004;93(8):10021006.

22. Radwan H, Selem A, Ghazal K. Value of N-terminal pro brain natriuretic peptide in predicting prognosis and severity of coronary artery disease in acute coronary syndrome. J Saudi Heart Assoc. 2014;26(4):192-198. 